\title{
Effects of Vascular Endothelial Growth Factor (VEGF) on Dental Pulp Stem Cells (DPSC)
}

\author{
${ }^{1}$ Cale Forgues, ${ }^{2}$ Eric Mullins and ${ }^{2}$ Karl Kingsley \\ ${ }^{I}$ Department of Advanced Education in Orthodontics, University of Nevada, \\ Las Vegas-School of Dental Medicine, 1700 West Charleston, Las Vegas, Nevada, 89106, USA \\ ${ }^{2}$ Department of Biomedical Sciences, University of Nevada, \\ Las Vegas-School of Dental Medicine, 1001 Shadow Lane, Las Vegas, Nevada, 89106, USA
}

\author{
Article history \\ Received: 10-02-2019 \\ Revised: 12-02-2019 \\ Accepted: 28-05-2019 \\ Corresponding Author: \\ Karl Kingsley \\ Department of Advanced \\ Education in Orthodontics, \\ University of Nevada, Las \\ Vegas-School of Dental \\ Medicine, 1700 West \\ Charleston, Las Vegas, Nevada, \\ 89106, USA \\ Tel: (702)774-2623 \\ Email: Karl.Kingsley@unlv.edu
}

\begin{abstract}
Dental Pulp Stem Cells (DPSCs) are non-embryonic, mesenchymal stem cells that may have significant potential for therapeutic and regenerative biomedical applications. Studies of DPSC differentiation have demonstrated the potential to form many tissue types, including neural, osteogenic and vascular precursors using cytokines and growth factors, such as Vascular Endothelial Growth Factor (VEGF). Eight previously isolated Dental Pulp Stem Cell (DPSC) isolates were grown in culture and treated with VEGF to evaluate any effects on growth, viability or biomarker expression. Administration of VEGF at $10 \mathrm{ng} / \mathrm{mL}$ significantly inhibited growth in two rapidly dividing or rDT DPSC isolates, with no other measurable effects noted among the intermediate (iDT) or slow (sDT) growing DPSC isolates. In addition, administration of VEGF had no significant effects on viability of the SDT or iDT DPSC isolates, however, all three of the rapidly dividing or rDT DPSC isolates exhibited significantly increased viability. Finally, mRNA expression of osteogenic biomarkers Alkaline Phosphatase (ALP) and Dentin Sialophosphoprotein (DSPP) was observed among the rDT isolates with specific combinations of DPSC biomarkers expressed (NANOG in combination with Sox-2 or Oct-4 but not both). The results of these data suggest that VEGF administration may be sufficient to induce partial differentiation of DPSC isolates, although this may be dependent upon the MSC biomarker expression of the DPSCs. These preliminary data may further research into the potential for tissue regeneration and bioengineering.
\end{abstract}

Key words: Dental Pulp Stem Cell (DPSC), Vascular Endothelial Growth Factor (VEGF), Cellular Differentiation

\section{Introduction}

Dental Pulp Stem Cells (DPSCs) are non-embryonic, mesenchymal stem cells than can be obtained, isolated, cultured and cryopreserved with relative ease compared with other potential sources, which has driven recent scientific research into their potential for therapeutic applications (Ferro et al., 2014; Gronthos et al., 2011; Collart-Dutilleul et al., 2015). Harvested from the dental pulp of primary teeth, extracted teeth, or avulsed teeth, DPSCs are multi-potent stem cells that may be useful to facilitate advanced regenerative therapies (Kabir et al., 2014; Aurrekoetxea et al., 2015). These studies have provided a better overall understanding of the capabilities of Mesenchymal Stem Cells (MSCs) and DPSCs, with recent evidence demonstrating that differentiation potential may depend, in part, on the tissue of origin used in MSC harvesting (Masthan et al., 2013; Isobe et al., 2016; Hernández-Monjaraz et al., 2018).

Studies etaldone on DPSC differentiation have demonstrated the potential to form many tissue types, including neural, osteogenic and vascular precursors (Gonmanee et al., 2018; Kim et al., 2012; Zhang et al., 2016). Much progress has been made towards the in vitro and in vivo differentiation of DPSC towards specific cell lineages (Zhang et al., 2008; Kanafi et al., 2013). In fact, some evidence now suggests that individual growth factors, such as Vascular Endothelial 
Growth Factor (VEGF), may be sufficient to induce partial differentiation of DPSC-although this may be more dependent upon specific biomarkers or DPSC characteristics (D' Alimonte et al., 2011; Janebodin et al., 2013; Silva et al., 2017).

It has been demonstrated that DPSCs can be stimulated using VEGF through the canonical Wnt- $\beta$ catenin pathway into differentiating into blood vessels that resembled embryonic vasculogenesis, revealing the importance of this growth factor (VEGF) in angiogenesis as well as its potential for regenerative vasculogenesis (Zhang et al., 2016; 2008; Silva et al., 2017). However, the majority of studies to date have examined how the family of VEGF ligands act on specific tyrosine kinase receptors to create intracellular responses in differentiated vascular endothelial cells, while the cellular responses to and intracellular effects of, VEGF on various lineages of multipotent DPSCs remain relatively unknown (Aksel and Huang, 2017).

Differentiation potential and stem-ness may be linked with specific intracellular MSC biomarkers such as the expression of Sox-2, Oct-4 and NANOG, which have been found to be highly associated with the pluripotency of cells, including DPSC (Yang et al., 2017; Alraies et al., 2017; Ferro et al., 2012). The presence or absence of these biomarkers in cultured DPSCs may determine the ability of the isolates to differentiate and self-replicate (Martens et al., 2012; Xiao et al., 2014; Bakkar et al., 2017). Based upon this understanding, the primary objective of this study was to evaluate the effects of VEGF on several DPSC isolates and to further evaluate the expression of specific biomarkers that may indicate pluripotency, as well as differentiation.

\section{Methods}

\section{Study Approval}

The protocol for this study was reviewed and approved by the Office for the Protection of Research Subjects (OPRS) and Institutional Review Board (IRB) OPRS\#763012-1 "Retrospective analysis of Dental Pulp Stem Cells (DPSC) from the University of Nevada Las Vegas (UNLV) School of Dental Medicine (SDM) pediatric and adult clinical population". The original protocol for the collection and isolation of DPSC was approved by the IRB and OPRS\#0907-3148 "Isolation of Non-Embryonic Stem Cells from Dental Pulp".

\section{Study Design}

This retrospective study involved the analysis of DPSCs previously isolated from clinical patients, recruited at random from the UNLV-SDM pediatric clinic. Inclusion criteria included adult patients or pediatric patients aged seven (7) or older with their parents or guardian's permission who agreed to participate and were scheduled for a tooth extraction of health (vital) intact teeth prior to the initiation of orthodontic treatment. Pediatric assent and Parental permission to consent for voluntary participation were obtained at the time of study enrollment. Exclusion criteria included any patient, parent or child that was not a patient of record at UNLV-SDM, any patient or guardian who declined to participate and any patients having teeth extracted due to injury (fracture), infection or other disease.

\section{DPSC Collection (Initial)}

In brief, the overwhelming majority of patients who agreed to participate were scheduled for tooth extractions of third molars. Once extracted, each tooth was sectioned at the Cemento-Enamel Junction (CEJ) to allow extraction of the dental pulp with an endodontic broach for transfer into a sterile microcentrifuge tube containing $1 \mathrm{X}$ Phosphate-Buffered Saline (PBS).

Samples were stored on ice until transfer to a biomedical laboratory for processing and screening. To prevent research bias and prevent any patient identifying information from being disclosed, a randomly generated, non-duplicated number was assigned to each sample and concurrent patient demographic information collected. No patient-specific identifying information was subsequently available to any research team member.

\section{Culture and Propagation}

Briefly, cells were cultured and propagated for ten passages to determine the rate of growth and Doubling Time (DT). Passage (or split) for each DPSC isolate was 1:2 and confluence determined with trypan blue and BioRad TC20 automated cell counter (Hercules, $\mathrm{CA}$ ), using the manufacturer recommended protocol. Data collected included total and live cell number and the resulting percentage of viable cells for analysis. Doubling Time (DT) was categorized as rapid or rDT ( $\sim 2$ days), intermediate or iDT (4-6 days) and slow or sDT (10-12 days).

\section{Experimental Protocol}

To determine any effects on DPSCs, the cells were plated into 96-well tissue culture treated plates at a concentration of $1.2 \times 10^{4}$ cells $/ \mathrm{mL}$. Negative (nontreated) control cells were compared with cells treated with Vascular Endothelial Growth Factor (VEGF) from ThermoFisher Scientific (PCH9394) at a concentration of $10 \mathrm{ng} / \mathrm{mL}$. Eight replicates were performed in each experiment for all DPSC isolates, which were repeated for a total of three experimental trials $(n=24)$.

\section{$R N A$ Isolation}

To assess any changes to differentiation, total RNA was isolated from each isolate using the Total RNA 
Isolation Reagent (TRIR) from Molecular Research Center (Cincinnati, $\mathrm{OH}$ ) using the protocol recommended by the manufacturer. RNA was subsequently screened for quality and quantity using ratio measurements of absorbance at 260 and $280 \mathrm{~nm}$ (A260/A280 ratio).

\section{Polymerase Chain Reaction (PCR)}

Screening for changes to mRNA expression in each DPSC isolated was accomplished using the ABgene Reverse-iT One-Step RT-PCR protocol and reagent kit with specifications that included an initial reverse transcription at $47 \mathrm{C}$ for $30 \mathrm{~min}$, followed by $30 \mathrm{dpsc}-$ 3882 , annealing for $30 \mathrm{sec}$ at the appropriate temperature for each primer set and final extension at $60 \mathrm{C}$ for one minute. Primers synthesize from Eurofins MWG Operon (Huntsville, AL) were:

Glyceraldehyde 3-phosphate dehydrogenase (GAPDH); Annealing temperature 67C

Forward primer-GAPDH,

ATCTTCCAGGAGCGAGATCC; 20 nt, 55\% GC, Tm 66C

Reverse primer-GAPDH, ACCACTGACACGTTGGCAGT; 20 nt, 55\% GC, Tm 70C

Optimal temperature T(opt): Lower temperature $-5 \mathrm{C}=61 \mathrm{C}$

Alkaline phosphatase (ALP); Annealing temperature: $72 \mathrm{C}$

Forward primer-ALP, CACTGCGGACCATTCCCACGTCTT;24 nt, $58 \%$ GC, Tm 74C

Reverse primer- ALP,
GCGCCTGGTAGTTGTTGTGAGCAT; 24 nt, 54\% GC, Tm 72C

Optimal temperature $\mathrm{T}(\mathrm{opt}) \mathrm{L}$ Lower temperature $-5 \mathrm{C}=67 \mathrm{C}$

Dentin sialophosphoprotein (DSPP); Annealing temperature: $68 \mathrm{C}$

Forward primer-DSPP, CAACCATAGAGAAAGCAAACGCG;23 nt, $48 \%$ GC, Tm 67C

Reverse primer- DSPP, TTTCTGTTGCCACTGCTGGGAC; 22 nt, $55 \%$ GC, Tm 70C

Optimal temperature T(opt): Lower temperature $-5 \mathrm{C}=62 \mathrm{C}$

\section{Statistical Analysis}

Basic proliferation and viability information regarding the DPSC isolated were compiled and presented using simple descriptive statistics (counts and percentages). Comparisons of change to viability or proliferation were calculated and compared using two- tailed t-tests, which are appropriate for parametric data analysis. Due to the potential for Type I error, all analyses were subsequently confirmed using analysis of variance or multiple (ANOVA).

\section{Results}

To determine any effects on DPSC phenotypes, vascular endothelial growth factor (VEGF) was administered in 96-well assays (Fig. 1). These results demonstrated that the majority of DPSC isolates were not significantly affected by VEGF administration, $p>0.05$. However, two DPSC isolates (dpsc-3882, dpsc-5653) had significant measurable decreases in proliferation under VEGF administration, $p=0.038$ and $p=0.041$ respectively. In addition, dpsc-3882 and dpsc-5653 were both categorized as having rapid doubling times or $\mathrm{rDT}$.

To evaluate if the observed changes in proliferation and cellular growth correlated with any changes to other DPSC phenotypes, cellular viability was also measured under VEGF administration (Fig. 2). Although no significant changes to viability were noted among the iDT or SDT DPSC isolates under VEGF administration, all three of the rDT DPSC isolates demonstrated significant measurable increases to viability over the 72 time course, $p<0.05$.

To determine if any of the changes to cellular growth or viability induced by VEGF administration among the DPSC isolates were associated with changes to DPSC biomarkers for osteoblastic differentiation, RT-PCR screening of RNA was performed (Fig. 3). In brief, primers specific for Alkaline Phosphatase (ALP) and Dentin Sialophosphoprotein (DSPP) were used to screen for mRNA expression of these biomarkers. These results demonstrated that VEGF administration was sufficient to induce mRNA expression of ALP in two DPSC isolates (dpsc-3882, dpsc-5653). In addition, VEGF administration was also sufficient to induce DSPP mRNA expression in one DPSC isolate (dpsc-3882).

Finally, an evaluation of the MSC biomarkers for each DPSC isolate was performed to determine if there were any associations with VEGF responsiveness (Fig. 4). This analysis revealed that MSC biomarkers Sox-2, Oct4 and NANOG were differentially expressed by the DPSC isolates (Fig. 4A). For example, the rDT DPSC isolates each had a distinct expression profile (dps-3882: Oct-4, NANOG; dpsc-5653: Sox-2, NANOG; dpsc7089: Sox-2, Oct-4, NANOG). In contrast, none of the iDT DPSC isolates expressed Oct-4, while none of the sDT expressed either Sox-2 or Oct-4.

When combined with the results of VEGF assay, these data demonstrated that only the rDT DPSC isolates that expressed a combination of NANOG with either Oct-4 or Sox-2 (but not both) were responsive to VEGF administration (Fig. 4B). More specifically, the rDT DPSC isolate expressing a combination of Oct- 4 and 
NANOG exhibited the most robust VEGF response, producing both ALP and DSPP (dpsc-3882). The rDT DPSC isolate expressing the combination of Sox-2 and NANOG exhibited some response to VEGF, producing ALP but not DSPP (dpsc-5653). However, the rDT isolate that expressed all three MSC biomarkers (Sox-2, Oct-4, NANOG) did not exhibit an osteogenic response to VEGF administration-similar to the negative response of the iDT DPSC isolates (Sox-2, NANOG) and sDT DPSC isolates (NANOG only).

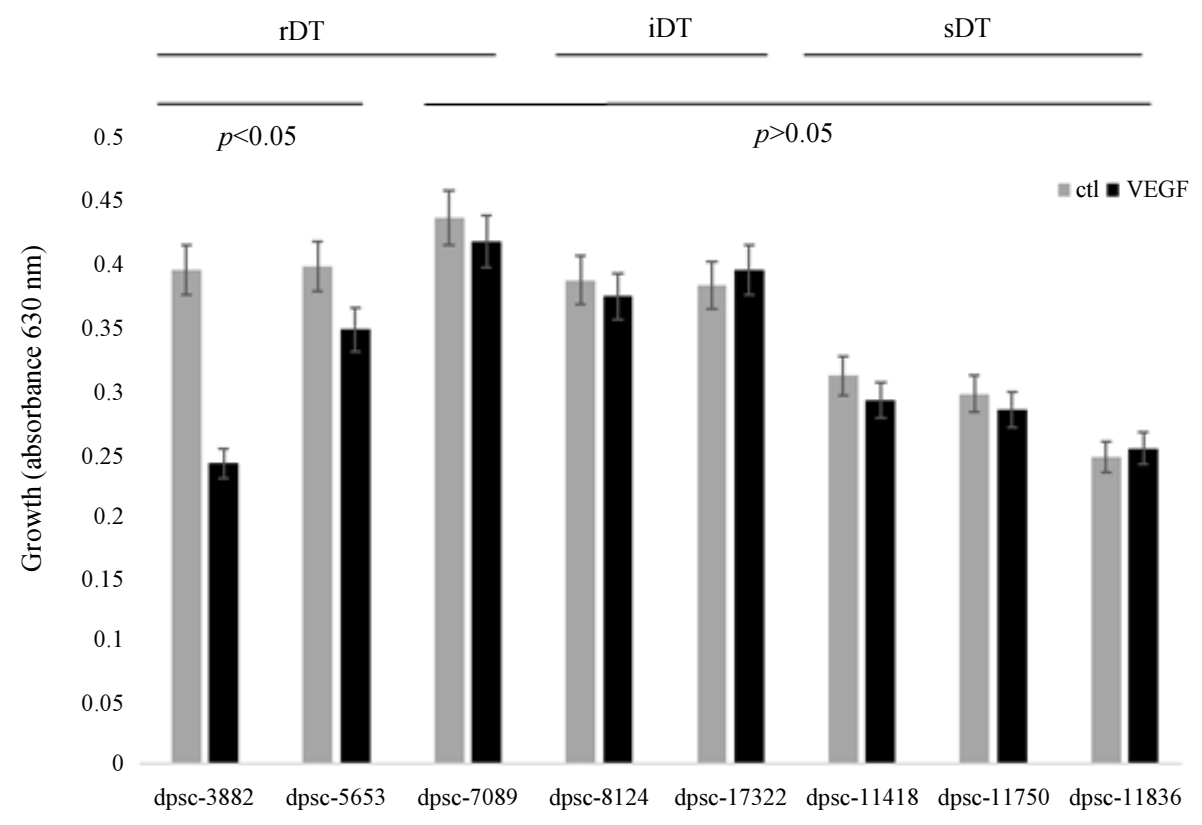

Fig. 1: Effects of VEGF administration on DPSC growth. Administration of VEGF at $10 \mathrm{ng} / \mathrm{mL}$ had a significant effect on two rapidly dividing (rDT) DPSC isolates, dpsc-3882 and dpsc-5653-which were significantly lower than the negative controls $(p=0.038$ and $p=0.041$, respectively). No other measurable effects were noted among the intermediate (iDT) or slow (sDT) DPSC isolates

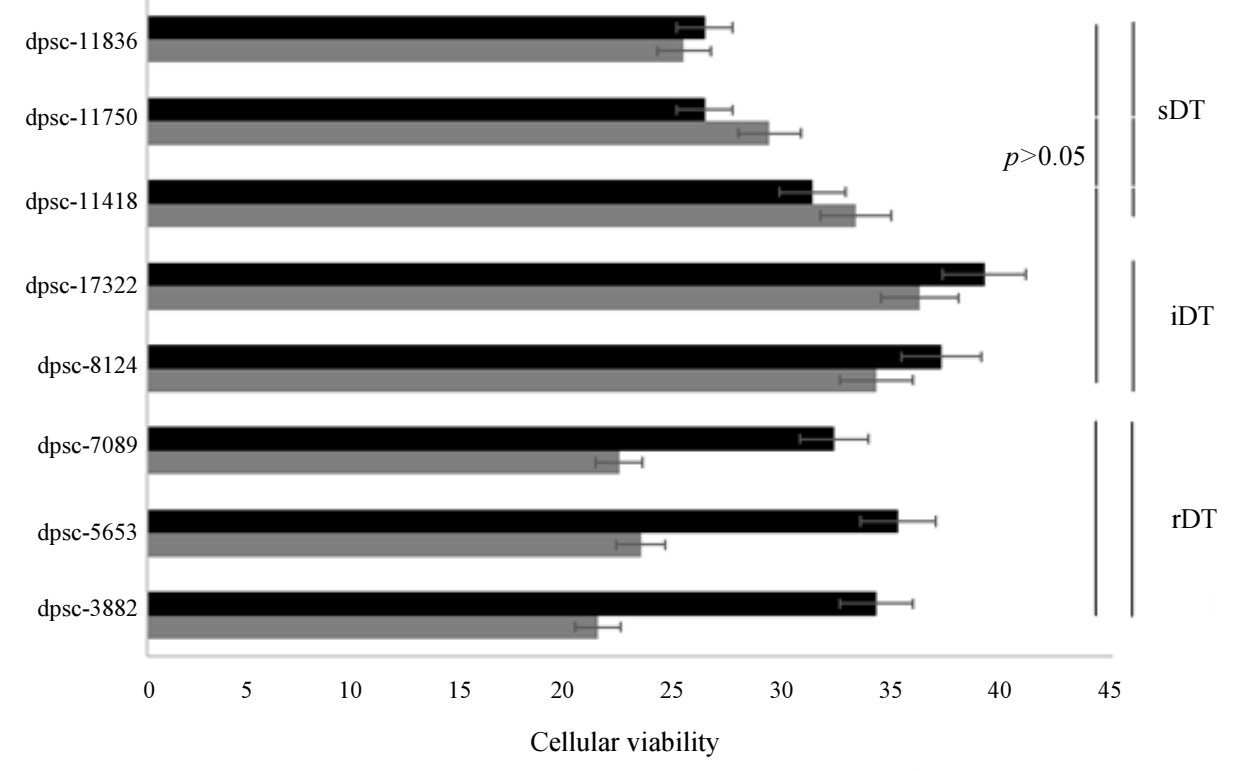

Fig. 2: Effects of VEGF administration on DPSC viability. Administration of VEGF at $10 \mathrm{ng} / \mathrm{mL}$ had no significant effects on the intermediate (iDT) or slow (sDT) DPSC isolates, $\mathrm{p}>0.05$. However, all three of the rapidly dividing or rDT DPSC isolates, dpsc-3882, dpsc-5653, dpsc-7089 exhibited increased viability ( $p=0.018, p=0.011, p=0.122$, respectively) 


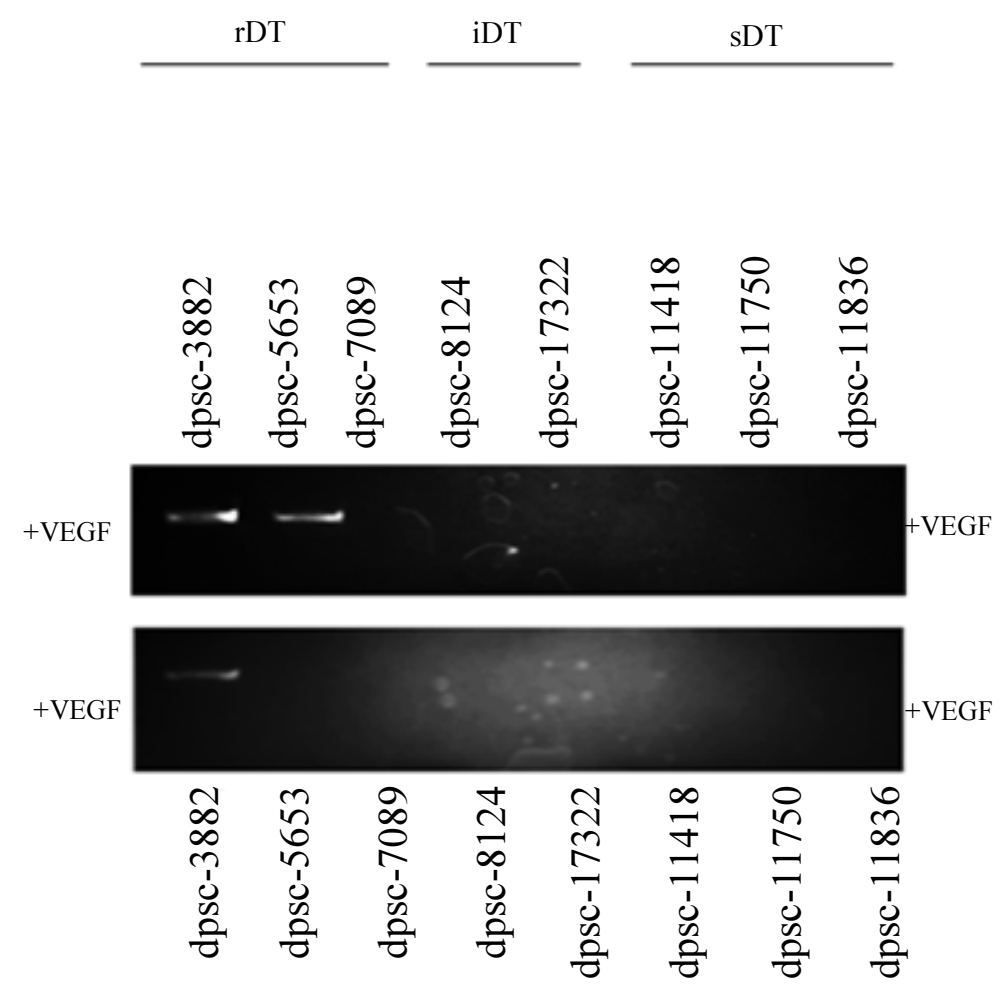

Fig. 3: DPSC osteogenic mRNA biomarker induction following VEGF treatment. VEGF administration (10 ng/mL) was sufficient to induce mRNA expression of Alkaline Phosphatase (ALP) in two rDT DPSC isolates (dpsc-3882, dpsc-5653) and Dentin Sialophosphoprotein (DSPP) mRNA expression in dpsc3882. Expression of these mRNA biomarkers were not evident in any of the other DPSC isolates

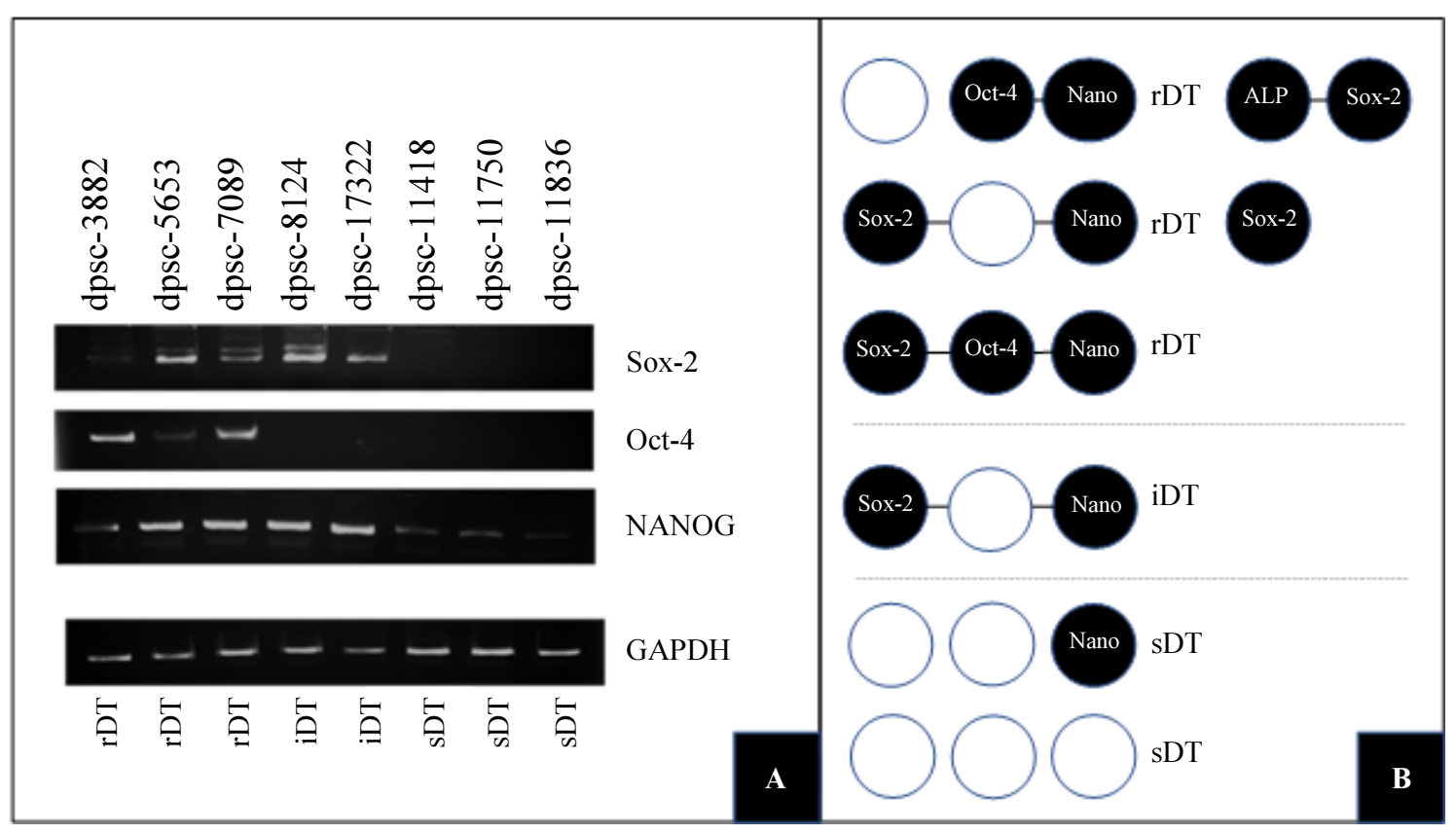

Fig. 4: MSC mRNA biomarker analysis of DPSC isolates. Osteogenic VEGF response was observed in DPSC isolates expressing NANOG in combination with either Oct-4 (dpsc-3882) or Sox-2 (dpsc-5653) but not both (dpsc-7089). No osteogenic VEGF response was noted among the iDT (Sox-2, NANOG) or sDT (NANOG) DPSC isolates 


\section{Discussion}

Research that has evaluated DPSC differentiation has demonstrated the potential to form many cell types, including neural, osteogenic and vascular precursors using cytokines and growth factors, such as vascular endothelial growth factor (Zhang et al., 2016; 2008). However, the phenotypic and cellular effects of VEGF on various lineages of multipotent DPSCs remains relatively unknown, therefore this study sought to evaluate these effects on several DPSC isolates with distinct markers of pluripotency (D' Alimonte et al., 2011; Janebodin et al., 2013; Silva et al., 2017). The results of this study demonstrated that VEGF has distinct and specific effects on DPSC phenotypes, although these were not observed uniformly among all DPSC isolates.

For example, cellular growth and viability were markedly affected by VEGF only among the rapidly growing (rDT) DPSC isolates, which mirrors similar findings of VEGF effects on rapidly dividing MSC from other tissues (Chen et al., 2018; Healy et al., 2015; Yuan et al., 2011). In addition, VEGF appears to induce osteogenic biomarker expression in a subset of rDT DPSC isolates, a finding that appears to support observations of VEGF osteogenic effects in other MSCs (Zavan et al., 2017; Leegwater et al., 2017; Murakami et al., 2017). To understand these observations more thoroughly, an analysis of MSC biomarker expression and the associations with osteogenic marker induction may be necessary (Bakkar et al., 2017; Bakopoulou et al., 2017; Karamzadeh et al., 2012).

New evidence has suggested that MSC biomarker expression in DPSCs may determine, in part, their differentiation potential and responsiveness to external stimuli (Xie et al., 2018; Zhang et al., 2017; Xie et al., 2017). The results of this study support these findings, with observations that rapidly dividing DPSC isolates expressing NANOG in combination with either Sox-2 or Oct-4 were responsive to VEGF administration. This research may also provide a potential explanation for the observation that DPSC expression of Oct-4, Sox-2 and NANOG were not responsive to VEGF administration, noting that "stemness" and pluripotency are correlated with MSC biomarker expression and that DPSC expression of more MSC biomarkers may indicate more than one stimulus or induction factor may be needed to facilitate differentiation (Pisal et al., 2018; Lee et al., 2017).

\section{Conclusion}

The results of these data suggest that VEGF administration may be sufficient to induce partial differentiation of DPSC isolates, although this may be dependent upon the MSC biomarker expression of the DPSCs. In addition, the phenotypic changes to these DPSC isolates (decreased growth, increased viability) support these observations and may provide preliminary data to further research into the potential for osteogenic differential of DPSC. This may contribute to the overall, long-term goals of DPSC use for tissue regeneration and bioengineering.

\section{Acknowledgement}

Dr. Kingsley and Dr. Forgues would like to thank the Department of Advanced Education in Orthodontics and Dentofacial Orthopedics at the University of Nevada, Las Vegas, School of Dental Medicine for research funds to complete this pilot study.

\section{Conflicts of Interest}

The authors declare there are no conflicts of interest to report.

\section{Author Contributions}

Cale Forgues: Responsible for the sample processing and data collection, data analysis and manuscript preparation.

Eric Mullins: Responsible for the sample processing and data collection.

Karl Kingsley: Responsible for the data analysis and manuscript preparation.

\section{References}

Aksel, H. and G.T. Huang, 2017. Combined effects of vascular endothelial growth factor and bone morphogenetic protein 2 on odonto/osteogenic differentiation of human dental pulp stem cells in vitro. J. Endod., 43: 930-935.

Alraies, A., N.Y. Alaidaroos, R.J. Waddington, R. Moseley and A.J. Sloan, 2017. Variation in human dental pulp stem cell ageing profiles reflect contrasting proliferative and regenerative capabilities. BMC Cell Biol., 18: 12.

Aurrekoetxea, M., P. Garcia-Gallastegui, I. Irastorza, J. Luzuriaga and V. Uribe-Etxebarria et al., 2015. Dental pulp stem cells as a multifaceted tool for bioengineering and the regeneration of craniomaxillofacial tissues. Front Physiol., 6: 289.

Bakkar, M., Y. Liu, D. Fang, C. Stegen and X. Su et al., 2017. A simplified and systematic method to isolate, culture andcharacterize multiple types of human dental stem cells from a single tooth. Met. Mol. Biol., 1553: 191-207.

Bakopoulou, A., D. Apatzidou, E. Aggelidou, E. Gousopoulou and G. Leyhausen et al., 2017. Isolation and prolonged expansion of oral mesenchymal stem cells under clinical-grade, GMPcompliant conditions differentially affects "stemness" properties. Stem Cell Res Ther., 8: 247-247. 
Chen, L., W. Xia and M. Hou, 2018. Mesenchymal stem cells attenuate doxorubicin-induced cellular senescence through the VEGF/Notch/TGF- $\beta$ signaling pathway in $\mathrm{H} 9 \mathrm{c} 2$ cardiomyocytes. Int. J. Mol. Med., 42: 674-684.

Collart-Dutilleul, P.Y., F. Chaubron, J. De Vos and F.J. Cuisinier, 2015. Allogenic banking of dental pulp stem cells for innovative therapeutics. World J. Stem Cells, 7: 1010-21.

D' Alimonte, I., E. Nargi, F. Mastrangelo, G. Falco and P. Lanuti et al., 2011. Vascular endothelial growth factor enhances in vitro proliferation and osteogenic differentiation of human dental pulp stem cells. J. Biol. Regul. Homeost. Agents, 25: 57-69.

Ferro, F., R. Spelat and C.S. Baheney, 2014. Dental Pulp Stem Cell (DPSC) isolation, characterization and differentiation. Methods Mol. Biol., 1210: 91-115.

Ferro, F., R. Spelat, F. D'Aurizio, E. Puppato, M. Pandolfi and A.P. Beltrami et al., 2012. Dental pulp stem cells differentiation reveals new insights in Oct4A dynamics. PLoS One, 7: e41774.

Gonmanee, T., C. Thonabulsombat, K. Vongsavan and H. Sritanaudomchai, 2018. Differentiation of stem cells from human deciduous and permanent teeth into spiral ganglion neuron-like cells. Arch. Oral Biol., 88: 34-41.

Gronthos, S., A. Arthur, P.M. Bartold and S. Shi, 2011. A method to isolate and culture expand human dental pulp stem cells. Methods Mol. Biol., 698: 107-21.

Healy, M.E., R. Bergin, B.P. Mahon and K. English, 2015. Mesenchymal stromal cells protect against caspase 3-mediated apoptosis of CD19 $(+)$ peripheral B cells through contact-dependent upregulation of VEGF. Stem Cells Dev., 24: 2391-402.

Hernández-Monjaraz, B., E. Santiago-Osorio, A. Monroy-García, E. Ledesma-Martínez and V.M. Mendoza-Núñez, 2018. Mesenchymal stem cells of dental origin for inducing tissue regeneration in periodontitis: A mini-review. Int. J. Mol. Sci., 19: E944- E944.

Isobe, Y., N. Koyama, K. Nakao, K. Osawa and M. Ikeno et al., 2016. Comparison of human mesenchymal stem cells derived from bone marrow, synovial fluid, adult dental pulp andexfoliated deciduous tooth pulp. Int. J. Oral Maxillofac. Surg., 45: 124-31.

Janebodin, K., Y. Zeng, W. Buranaphatthana, N. Ieronimakis and M. Reyes, 2013. VEGFR2dependent angiogenic capacity of pericyte-like dental pulp stem cells. J. Dent. Res., 92: 524-31.

Kabir, R., M. Gupta, A. Aggarwal, D. Sharma and A. Sarin et al., 2014. Imperative role of dental pulp stem cells in regenerative therapies: A systematic review. Niger J. Surg., 20: 1-8.
Kanafi, M.M., A. Ramesh, P.K. Gupta and R.R. Bhonde, 2013. Influence of hypoxia, high glucose andlow serum on the growth kinetics of mesenchymal stem cells from deciduous and permanent teeth. Cells Tissues Organs, 198: 198-208.

Karamzadeh, R., M.B. Eslaminejad and R. Aflatoonian, 2012. Isolation, characterization and comparative differentiation of human dental pulp stem cells derived from permanent teeth by using two different methods. J. Vis. Exp., (69). pii: 4372.

Kim, B.C., H. Bae, I.K. Kwon, E.J. Lee and J.H. Park et al., 2012. Osteoblastic/cementoblastic and neural differentiation of dental stem cells and their applications to tissue engineering and regenerative medicine. Tissue Eng. Part B Rev., 18: 235-44.

Lee, S.H., A. Inaba, N. Mohindroo, D. Ganesh and C.E. Martin et al., 2017. Three-dimensional sphereforming cells are unique multipotent cell population in dental pulp cells. J. Endod., 43: 1302-1308.

Leegwater, N.C., A.D. Bakker, J.M. Hogervorst, P.A. Nolte and J. Klein-Nulend, 2017. Hypothermia reduces VEGF-165 expression, but not osteogenic differentiation of human adipose stem cells under hypoxia. PLoS One, 12: e0171492.

Martens, W., E. Wolfs, T. Struys, C. Politis and A. Bronckaers et al., 2012. Expression pattern of basal markers in human dental pulp stem cells and tissue. Cells Tissues Organs, 196: 490-500.

Masthan, K.M.K., S.L. Sankari, N.A. Babu and T. Gopalakrishnan, 2013. Mystery inside the tooth: The dental pulp stem cells. J. Clin. Diagnostic Res., 7: 945-947.

Murakami, J., M. Ishii, F. Suehiro, K. Ishihata and N. Nakamura et al., 2017. Vascular endothelial growth factor-C induces osteogenic differentiation of human mesenchymal stem cells through the ERK and RUNX2 pathway. Biochem. Biophys. Res. Commun., 484: 710-718.

Pisal, R.V., J. Suchanek, R. Siller, T. Soukup and H. Hrebikova et al., 2018. Directed reprogramming of comprehensively characterized dental pulp stem cells extracted from natal tooth. Sci. Rep., 8: 6168-6168.

Silva, G.O., Z. Zhang, C. Cucco, M. Oh and C.H.R. Camargo et al., 2017. Lipoprotein receptor-related protein 6 signaling is necessary for vasculogenic differentiation of human dental pulp stem cells. J. Endod., 43: S25-S30.

Xiao, L., Y. Kumazawa and H. Okamura, 2014. Cell death, cavitation and spontaneous multidifferentiation of dental pulp stem cells-derived spheroids in vitro: A journey to survival and organogenesis. Biol Cell, 106: 405-19.

Xie, H., M. Chua, I. Islam, R. Bentini and T. Cao et al., 2017. CVD-grown monolayer graphene induces osteogenic but not odontoblastic differentiation of dental pulp stem cells. Dent. Mater., 33: e13-e21. 
Xie, H., N. Dubey, W. Shim, C.J.A. Ramachandra and K.S. Min, 2018. Functional odontoblastic-like cells derived from human iPSCs. J. Dent. Res., 97: 77-83.

Yang, Y., Y. Zhao, X. Liu, Y. Chen and P. Liu et al., 2017. Effect of SOX2 on odontoblast differentiation of dental pulp stem cells. Mol. Med. Rep., 16: 9659-9663.

Yuan, L., M.J. Wu, H.Y. Sun, J. Xiong and Y. Zhang et al., 2011. VEGF-modified human embryonic mesenchymal stem cell implantation enhances protection against cisplatin-induced acute kidney injury. Am. J. Physiol. Renal. Physiol., 300: F207- F218.

Zavan, B., L. Ferroni, C. Gardin, S. Sivolella and A. Piattelli et al., 2017. Release of VEGF from dental implant improves osteogenetic process: Preliminary in vitro tests. Materials (Basel), 10: E1052.
Zhang, W., X.F. Walboomers, T.H. Van Kuppevelt, W.F. Daamen and P.A. Van Damme et al., 2008. In vivo evaluation of human dental pulp stem cells differentiated towards multiple lineages. J. Tissue Eng. Regen. Med., 2: 117-25.

Zhang, X., H. Li, J. Sun, X. Luo and H. Yang et al., 2017. Cell-derived micro-environment helps dental pulp stem cells promote dental pulp regeneration. Cell Prolif.

Zhang, Z., F. Nör, M. Oh, C. Cucco and S. Shi et al., 2016. Wnt/ $\beta$-catenin signaling determines the vasculogenic fate of postnatal mesenchymal stem cells. Stem Cells. DOI: 10.1002/stem.2334 